\title{
CONF-970656--
}

\section{PHOTOREFRACTIVE LASER ULTRASOUND SPECTROSCOPY FOR MATERIALS CHARACTERIZATION}

\author{
K.L. Telschow, V.A. Deason, K.L. Ricks and R. S. Schley \\ Idaho National Engineering and Environmental Laboratory \\ Lockheed Martin Idaho Technologies Company \\ Idaho Falls, ID 83415-2209 \\ 1001231937 \\ 0911
}

\section{INTRODUCTION}

Ultrasonic elastic wave motion is often used to measure or characterize material properties. Through the years, many optical techniques have been developed for applications requiring noncontacting ultrasonic measurement. Most of these methods have similar sensitivities and are based on time domain processing using interferometry ${ }^{1}$. Wide bandwidth is typically employed to obtain real-time surface motion under transient conditions. However, some applications, such as structural analysis, are well served by measurements in the frequency domain that record the randomly or continuously excited vibrational resonant spectrum. A significant signal-to-noise ratio improvement is achieved by the reduced bandwidth of the measurement at the expense of measurement speed compared to the time domain methods. Complications often arise due to diffuse surfaces producing speckle that introduces an arbitrary phase component onto the optical wavefront to be recorded. Methods that correct for this effect are actively being investigated today.

Adaptive interferometry, which utilizes the photorefractive effect in optically nonlinear materials, offers a potentially powerful method to automatically correct for environmental and speckle effects. ${ }^{2,3}$ Photorefractivity employs optical excitation and transport of charge carriers to produce a hologram of the interference pattern developed inside the material. A spatially and temporally modulated charge carrier distribution results that is a direct measure of the phase information impressed onto the optical object beam by the vibrating surface. This hologram stores phase information from all the surface points on the vibrating specimen simultaneously. The hologram developed then can be detected through diffraction of a readout beam off this photoinduced volume grating. Several optical frequency domain measurement methods of vibration have been proposed using photorefractive two-wave and four-wave mixing in select materials. ${ }^{4,5,6}$ These have provided a response that is a nonlinear function of the specimen vibration displacement amplitude. 


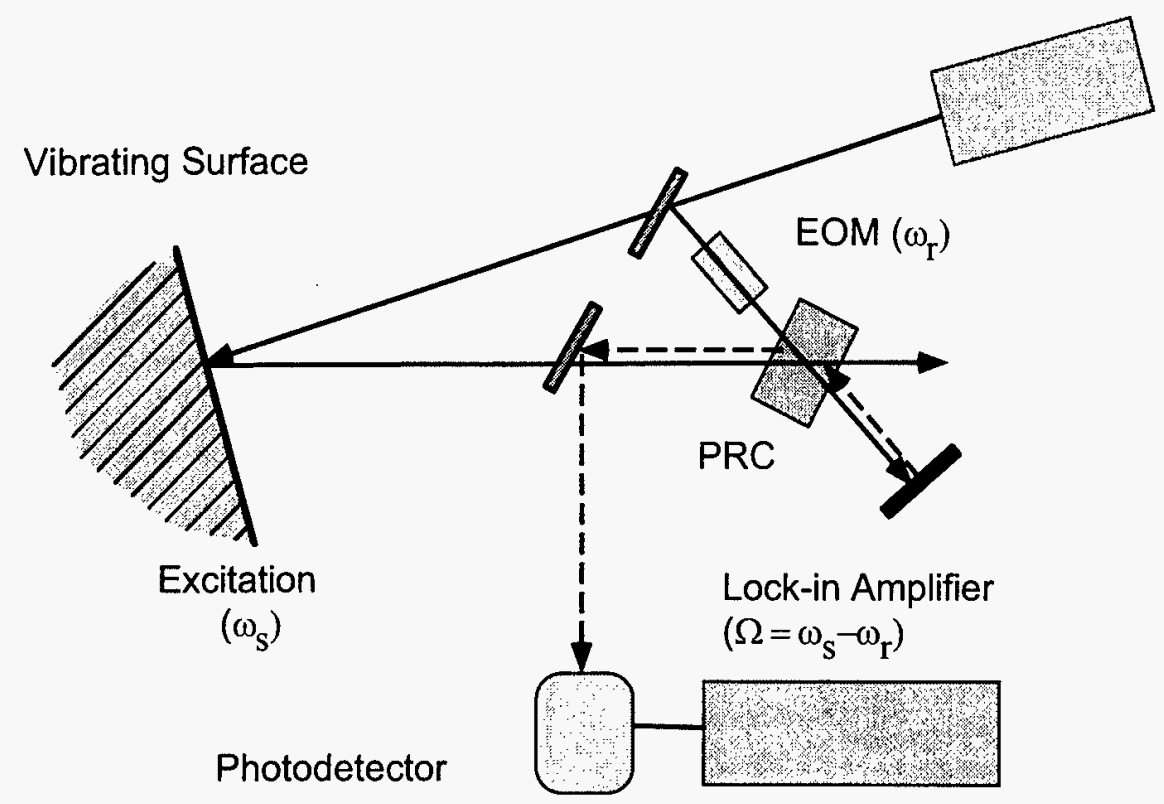

Figure 1. Photorefractive detection of resonant vibration, BS (beamsplitter), EOM (electro-optic modulator), (PRC) photorefractive crystal.

Recently, a method was developed for vibration detection ${ }^{7,8}$ that employs the photorefractive effect in an optical lock-in synchronous detection manner." This method phase modulates the object and reference beams such that a moving photorefractive grating at a fixed beat frequency is established within the material regardless of the specimen vibration frequency. The intensity of a readout beam scattering off the photoinduced grating is a direct measure of the vibrational amplitude and phase. It can be used for spectral analysis with a response proportional to the Bessel function of order one, providing a linear output for small amplitudes. The method accommodates rough surfaces and exhibits a flat frequency response above the photorefractive cutoff frequency. A minimum detectable displacement of $2 \times 10^{-3} \mathrm{~nm}$ has been recorded; however, the limitation was from optical phase noise, not shot noise, suggesting that further improvement is possible. The corresponding demonstrated detectability was $4.5 \times 10^{-6} \mathrm{~nm}(\mathrm{~W} / \mathrm{Hz})^{1 / 2}$, in terms of the light power received at the photodiode.

In this paper, the ability of the optical lock-in process to provide absolute calibration of measured displacement amplitude is described and application of the method to full-field imaging of vibrating square plates is presented. Typically, the nonphotorefractive interferometric methods do not allow imaging of more than one surface point at a time. However, since the photorefractive process records a volume hologram of all vibrating surface points simultaneously, imaging can be achieved. Both four-wave and two-wave mixing configurations have been employed for reading out the vibration-induced phase grating image. Results are presented for vibration modes of a free square stainless steel plate with diffusely reflecting surfaces that is driven piezoelectrically at one corner.

\section{PHOTOREFRACTIVE OPTICAL LOCK-IN METHOD}

Figure 1 shows the experimental setup for optical detection of a specimen undergoing continuous vibration. A diode-pumped Nd:YAG laser source (532 nm, 200 $\mathrm{mW}$ ) is split into object and reference beams. The excited vibrational modes of the specimen determine the frequency-dependent displacement amplitude of the surface, which 


\section{DISCLAIMER}

This report was prepared as an account of work sponsored by an agency of the United States Government. Neither the United States Government nor any agency thereof, nor any of their employees, makes any warranty, express or implied, or assumes any legal liability or responsibility for the accuracy, completeness, or usefulness of any information, apparatus, product, or process disclosed, or represents that its use would not infringe privately owned rights. Reference herein to any specific commercial product, process, or service by trade name, trademark, manufacturer, or otherwise does not necessarily constitute or imply its endorsement, recommendation, or favoring by the United States Government or any agency thereof. The views and opinions of authors expressed herein do not necessarily state or reflect those of the United States Government or any agency thereof. 


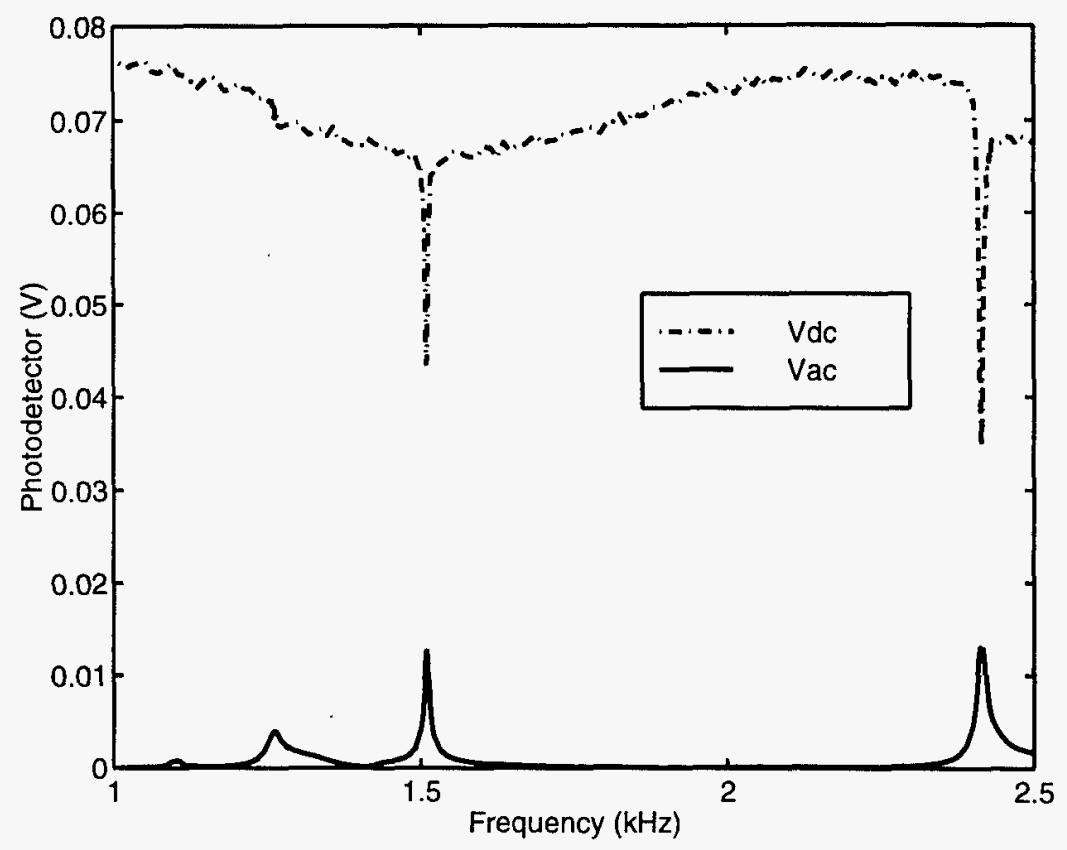

Figure 2. Photodetector $\mathrm{DC}$ and $\mathrm{AC}$ voltages from the vibration spectrum of two modes of a square stainless steel plate.

is transferred into phase modulation of the object beam. The reference beam is frequency modulated by an electro-optic modulator at a fixed modulation depth. The modulated beams are then combined and interfered inside a Bismuth Silicon Oxide (BSO) photorefractive crystal. A four-wave mixing configuration is used for readout of the photorefractive index grating for the single-point measurements. The reference beam is reflected back into the crystal along a counter-propagating path that matches the Bragg angle of the photorefractive grating in the medium. The resulting scattered wave is then sampled at the plate beamsplitter and deflected toward the photodetector. Subsequently, the photodetector signal is processed with conventional electrical lock-in methods to provide a measurement bandwidth of $1 \mathrm{~Hz}$. A recorded spectrum showing three spearate modes of a 1.5 inch square, 0.015 inch thick, stainless steel 304 plate is shown in figure 2; the plate is driven piezoelectrically at one corner.

A model of the lock-in process has been developed using one-dimensional plane wave coupled mode analysis. Both optical beams reaching the photorefractive crystal experience phase modulation due to the path lengths. In addition, the signal beam is modulated by the vibrating surface according to $\Phi_{s}=\Phi_{s 0} \sin \left(\omega_{s} t+\varphi_{s}\right), \quad \Phi_{s 0}=\frac{4 \pi \xi_{0}}{\lambda}$. The reference beam is phase modulated by the EOM according to $\Phi_{r}=\Phi_{r 0} \sin \left(\omega_{r} t+\varphi_{r}\right)$. The interference distribution and subsequent charge migration within the crystal generates a corresponding space charge electric field distribution, $E_{s c}$. The dynamic behavior of this field is controlled by the charge carrier mobility and trapping that produces, in the diffusive operation regime, a single relaxation time response given by

$$
\frac{\partial E_{s c}}{\partial t}+\frac{E_{s C}}{\tau}=\frac{i E_{q}}{\tau} \frac{\Delta I(\vec{r}, t)}{I_{0}}
$$




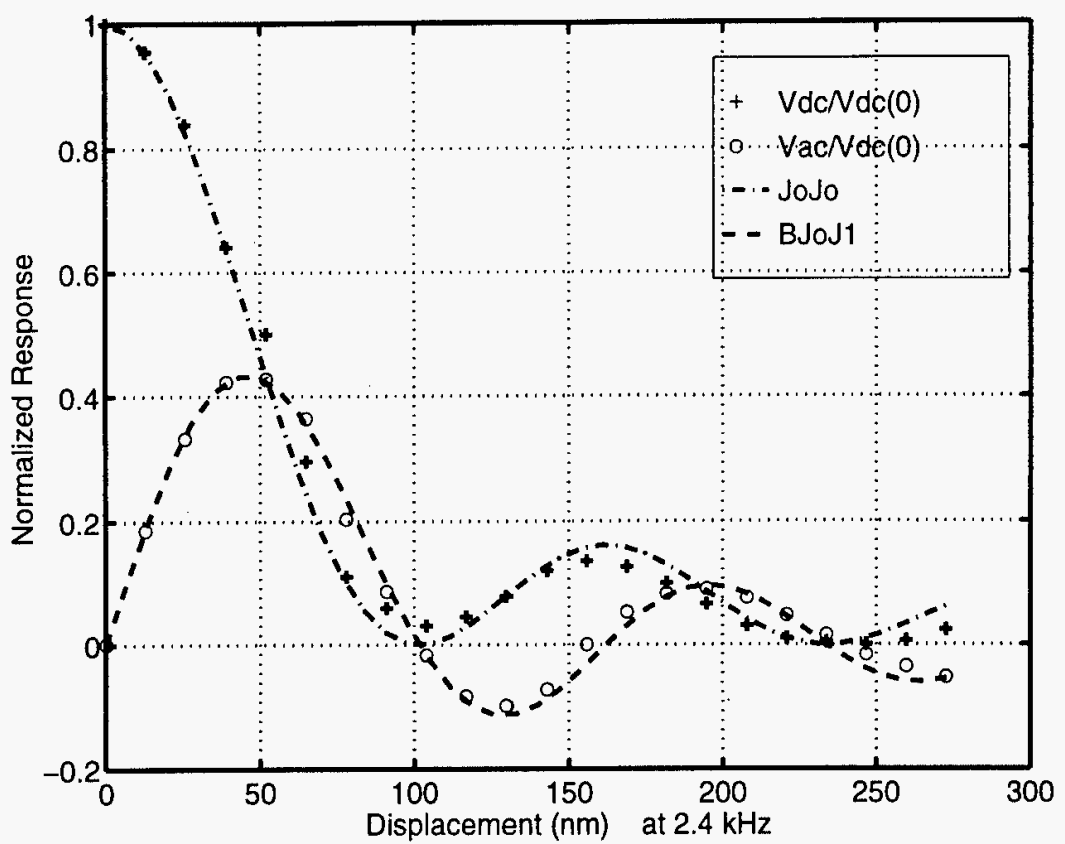

Figure 3. Measured DC and AC photodetector voltages for a square plate resonance as a function of the vibration amplitude.

where $\tau$ is the material response time, $\frac{\Delta I(\vec{r}, t)}{I_{0}}$ is the interference fringe contrast, and $E_{q}$ is the maximum achievable space-charge field, controlled by the concentration of available charge trapping sites and the fringe spacing. In the above configuration, the photorefractive crystal acts as a mixing and low-pass filtering element, providing the benefits of lock-in detection. Therefore, the space charge field responds to slowly varying phase modulations occurring within the material response time, allowing only the terms around the difference frequency $\Omega=\omega_{s}-\omega_{r}$ to be important. The space-charge field modulates the local refractive index through the linear electro-optic effect. This effect creates a diffraction grating within the crystal that contains the low frequency phase information desired. Several methods can be used to read out the space charge field and diffraction grating, including four-wave mixing, two-wave mixing with polarization selection, and electrical measurement through conduction of photoexcited carriers.

In the four-wave mixing arrangement, the reference beam that passes through the crystal is reflected back and diffracts into the signal beam direction, see figure 1 . The magnitude of the index of refraction grating produced is proportional to the space charge field and the orientation-dependent electro-optic coefficient in BSO. The diffracted beam intensity is a direct measure of the grating established and produces DC and AC output photodetector voltages given by

$$
\begin{aligned}
& \frac{\mathrm{V}_{\mathrm{DC}}\left(\Phi_{s 0}\right)}{\mathrm{V}_{\mathrm{DC} 0}}=\mathrm{J}_{0}^{2}\left(\Phi_{s 0}\right), \quad \mathrm{V}_{\mathrm{DC} 0}=\mathrm{V}_{\mathrm{DC}}\left(\Phi_{s 0}=0\right)=C \mathrm{~J}_{0}^{2}\left(\Phi_{r 0}\right) \\
& \frac{\mathrm{V}_{\mathrm{AC}}\left(\Phi_{s 0}\right)}{\mathrm{V}_{\mathrm{DC} 0}}=\mathrm{J}_{0}\left(\Phi_{s 0}\right) \mathrm{J}_{1}\left(\Phi_{s 0}\right)\left[\frac{\mathrm{J}_{1}\left(\Phi_{r 0}\right)}{\mathrm{J}_{0}\left(\Phi_{r 0}\right)} \frac{4}{\sqrt{1+\Omega^{2} \tau^{2}}}\right] \cos \left(\Omega \mathrm{t}+\Psi+\left(\varphi_{s}-\varphi_{r}\right)\right)
\end{aligned}
$$




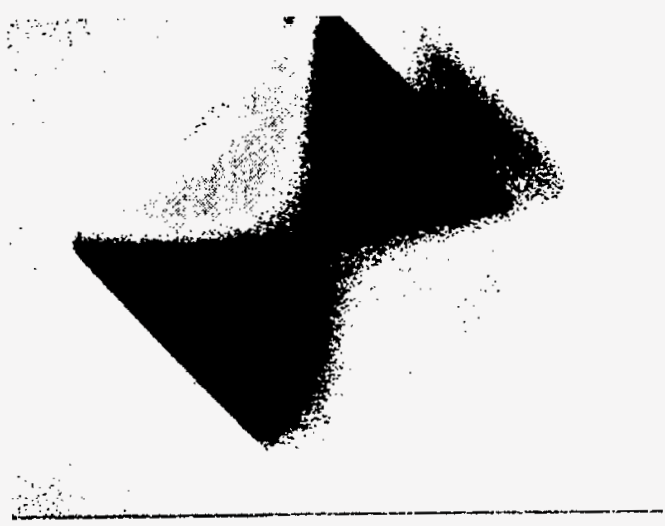

(a)

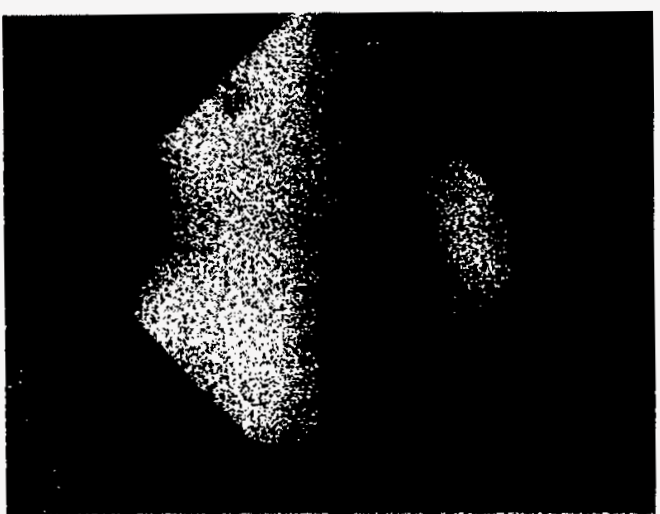

(b)

Figure 4. Vibrational mode images of a free square plate at (a) $1.5 \mathrm{kHz}$, (b) $2.4 \mathrm{kHz}$

where $\tan (\psi)=\Omega \imath$. Since the reference modulation and the photorefractive time constant are known, measurement of the $\mathrm{DC}$ and $\mathrm{AC}$ voltage magnitudes provides a direct calibration of the vibration amplitude in terms of the known optical wavelength through

$$
\frac{2 \mathrm{~J}_{1}\left(\Phi_{s 0}\right)}{\mathrm{J}_{0}\left(\Phi_{s 0}\right)}=\left|\frac{\mathrm{V}_{\mathrm{AC}}}{\mathrm{V}_{\mathrm{DC}}}\right| B\left(\Phi_{r 0}, \Omega \tau\right) \approx \Phi_{s 0}=\frac{4 \pi \xi_{0}(\omega)}{\lambda}, \text { for } \Phi_{s 0} \ll 1
$$

where $B\left(\Phi_{r 0}, \Omega \imath\right)$ is the controllable parameter given by the bracketed term in eq. 3 . The measured vibration amplitude dependence support this result as shown in figure 3 , which shows results from a single mode with $\Phi_{r 0} \approx 1.1$ and $\Omega \tau \approx 1$. In this figure, the vibration amplitude was determined by the best fit of the data to eq. 3 at small drive amplitudes. The overall consistency of the results supports the model and the calibration procedure.

\section{VIBRATION IMAGING}

A two-wave approach, based on polarization rotation through anisotropic selfdiffraction, ${ }^{10,1 !}$ was used for imaging as it offers improved light throughput from diffusely reflecting surfaces compared to the four-wave method. Figure 4 shows images of two modes of a free square stainless steel plate excited by a contact piezoelectric transducer at one corner. The nodal lines are clearly defined, and the relative phases of the vibration displacements are indicated by the light and dark areas. The entire modal pattern can be made to switch from light to dark by varying the offset frequency, $\Omega / 2 \pi$ (in this case, equal to $2 \mathrm{~Hz}$ ) between the object and reference excitations. This provides a powerful tool for visual mode searching and suggests processing methods that can be employed to enhance the detectability of specific modes. The minimum detectable displacement in the imaging mode $(\sim 1 \mathrm{~nm})$ is much larger than for the point detection method as no post electronic lockin processing was performed for the data of figure 4 . 


\section{CONCLUSIONS}

A photorefractive optical lock-in vibration spectral measurement method has been described that provides a simple method for quantitative determination of the vibration amplitude. The method uses optical synchronous or lock-in detection which can also include conventional electrical lock-in detection for narrow bandwidth high sensitivity measurements. A minimum detectable displacement of $2 \times 10^{-3} \mathrm{~nm}$ has been demonstrated using the single-point vibration detection method with a $1 \mathrm{~Hz}$ bandwidth. Readout methods employing four-wave or two-wave mixing produce an output intensity directly proportional to the amplitude of the vibration being measured, for amplitudes small compared with the optical wavelength, and provide the capability for mechanical phase measurement if synchronous excitation is used. Vibration imaging was shown from a diffusely scattering surface showing detectable displacements in the 1-45 nanometer range. The method is capable of flat frequency response over a wide range above the cutoff of the photorefractive effect and is applicable to rough surfaces.

\section{ACKNOWLEDGMENTS}

This work was supported through the INEEL Laboratory Directed Research \& Development program under DOE Idaho Operations Office Contract DE-AC0794ID13223.

\section{REFERENCES}

1 J. W. Wagner, "Optical Detection of Ultrasound," Physical Acoustics, Vol.XIX, Eds. Thurston, R.N., and Pierce, A.D., (Academic Press, New York, 1990) Chp. 5.

2 P. Yeh, Introduction to Photorefractive Nonlinear Optics, (John Wiley, New York, 1993).

3 S. I. Stepanov, International Trends inOptics, (Academic Press, New York, 1991) Chp. 9

4 J. P. Huignard and A. Marrakchi, "Two-wave mixing and energy transfer in $\mathrm{Bi}_{12} \mathrm{SiO}_{20}$ crystals: application to image amplification and vibration analysis," Opt. Lett., 6, (12), 622 (1981).

5 H. R. Hofmeister and A. Yariv, "Vibration detection using dynamic photorefractive gratings in KTN/KLTN crystals," Appl. Phys. Lett., 61 (20), 2395 (1992).

6 H. Rohleder, P. M. Petersen and A. Marrakchi, "Quantitative measurement of the vibrational amplitude and phase in photorefractive time-average interferometry: A comparison with electronic speckle pattern interferometry," J. Appl. Phys., 76 (1), 81 (1994).

7 T.C. Hale and K. Telschow, "Optical lock-in vibration detection using photorefractive frequency domain processing," Appl. Phys. Lett. 69, 2632 (1996).

8 T.C. Hale and K.L. Telschow, "Vibration modal analysis using all-optical photorefractive processing," Proc. SPIE Vol. 2849 , Photorefractive Fiber and Crystal Devices: Materials, Optical Properties, and Applications II, Francis T. Yu; Shizhuo Yin; Eds., p. 300 (1996).

9 J. Khoury, V. Ryan, C. Woods and M. Cronin-Golomb, "Photorefractive optical lock-in detector," Opt. Lett., 16, 1442 (1991).

10 R.C. Troth and J.C. Dainty, "Holographic interferometry using anisotropic self-diffraction in $\mathrm{Bi}_{12} \mathrm{SiO}_{20}$," Opt. Lett., 16 (1), 53 (1991).

11 T.C. Hale, K.L. Telschow and V.A. Deason, "Photorefractive optical lock-in vibration spectral measurement," submitted for publication to Applied Optics. 
M98050463

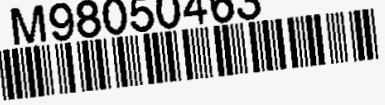

Report Number (14) $\frac{I N E L-96 / 00368}{\text { CONE-970656-- }}$

Dubl. Date (11) 199710

sponsor Code (18) DOE, XF

$J C$ Category (19) UC-900, DOEIER

DOE 